\title{
EL ZOOBENTOS DE LOS LAGOS Y EMBALSES ESPAÑOLES.
}

\section{Narcís Prat}

Dept. Ecologia, Univ. Barcelona, Diagonal, 645; 08028 BARCELONA.

Palabras clave: bentos, tipología, quironómidos, oligoquetos.

\section{ABSTRACT}

A summary is provided on the results of the study of sublittoral and profundal benthos of lakes and reservoirs in Spain. Oxygen is the key factor in determining the community in many reservoirs and in the karstic lake Banyoles. In Spanish lakes and reservoirs higher temperatures originate longer stratification periods and therefore longer periods without oxygen close to the bottom with the same amount of organic matter. This lack of oxygen with the abundance of sulphates in many water bodies makes the presence of sulphide common and for this reason many species are absent of the bottom of Spanish waters. Allocthonous organic matter inputs are important in our lakes and reservoirs and a key factor in small lakes like Banyoles and Sanabria and for this reason the organisms related to the meoifaunal loop (many of them microcarnivorous chironomids) are more important than phytophages. In addition to these factors, in reservoirs, water level fluctuation acts as a disturbance factor that prevents further organisation of communities. Thus many communities of Spanish lakes and reservoirs are dominated by meiofaunal elements (cladocera, nematoda, ostracoda) and its predators such as Procladius, Chaoborus, Cladopelma and Microchironomus. Oligochaeta are more abundant in the deeper zones, while Chironomus are more abundant in the shallower parts of reservoirs. The typological system of SAETHER is not applicable to Spanish lakes or reservoirs below $1500 \mathrm{~m}$ of altitude due to climatic differences between northern and southern European lakes, except in the case of Sanabria. This reduces its utility to the small lakes of the Pyrenees and the small ponds in Sierra Nevada mountains

\section{INTRODUCCION.}

En los últimos años hemos asístido a un avance lento pero constante del conocimiento de las comunidades que pueblan los fondos blandos de nuestros lagos y embalses, lo que llamamos el bentos de las zonas profundas o sublitorales. De complejidad relativamente simple, con densidades en muchos casos bajas y poblados por organismos de taxonomia complicada, estas comunidades no han recibido mucha atención por parte de los bentólogos españoles de aqui su escaso conocimiento. De hecho la mayoria de los trabajos se han realizado por parte del grupo de la Universidad de Barcelona que comenzó su estudio hace 20 años.

\section{AMBIENTES ESTUDIADOS.}

Historicamente el bentos de los embalses fué el primero en estudiarse por la conexión de su estudio al mas general de la limnología de nuestros embalses (Margalef, et al, 1976; Prat 1976, 1978, 1980b). En los embalses también se desarrollaron los primeros estudios de colonización en sustratos artificiales (Prat 1980c) así como la diferenciación entre faunas litorales, sublitorales y profundas (Prat, 1979b; Prat \& et al 1991). El estudio de embalses se ha repetido recientemente y sus primeros resultados se estan publicando actualmente (Real \& Prat, 1991). Existe asímismo un estudio algo 
mas detallado de un embalse, el de Talave (Murcia) (Malo, 1987 ) así como un trabajo excelente sobre 29 embalses de la Cuenca del Duero realizado por Limnos (1989) que seguramente es uno de los pocos trabajos de consultoría que trata el bentos con la profundidad necesaria.

España, como es conocido, es un pais sin grandes lagos aunque existe un rosario considerable de pequeños lagos y lagunas, especialmente en el Pirineo. De ellos los lagos cársticos han recibido un interés constante por parte de nuestros limnólogos desde hace tiempo, pero nunca se habia estudiado el bentos. Recientemente se ha abordado el estudio del bentos del lago de Banyoles, tanto de la zona sublitoral como de la profunda. Ello ha permitido caracterizar la comunidad (Rieradevall \& Prat, 1989, 1991) así como el estudio de los ciclos biológicos de algunos quironómidos y su producción (Prat \& Rieradevall, en prensa). Asímismo el estudio del lago de Banyoles ha permitido introducir algunas metodologías novedosas, como la clasíficación de los quironómidos por métodos citotaxonómicos.

En los lagos de montaña españoles, no se tienen para el bentos datos comparativos como los conocidos de los lagos franceses pirenaicos (Laville 1972). Tenemos alguna información indirecta de las comunidades de quironómidos de los lagos de Sierra Nevada por el trabajo de Laville \& Vilchez (1986). Recientemente hemos abordado el estudio del lago de Sanabria (Rieradevall et al en prensa) del cual teníamos solo algunos datos fraccionarios (Prat, 1980b).

Aun queda por iniciar el estudio de las comunidades bentónicas de dos grandes grupos de ambientes acuáticos: las de los lagos y lagunas de montaña (especialmente los de la parte centro y sur que posiblemente sean algo diferentes de los de los Pirineos) y las correspondientes a los humedales de las cuales casí no conocemos nada excepto algunos estudios relacionados con la alimentación de la anguila (Molina, 1989). Como resumen podemos decir que tenemos datos suficientes para 111 embalses, el lago de Banyoles, el de Sanabria y una laguna (Figura 1).

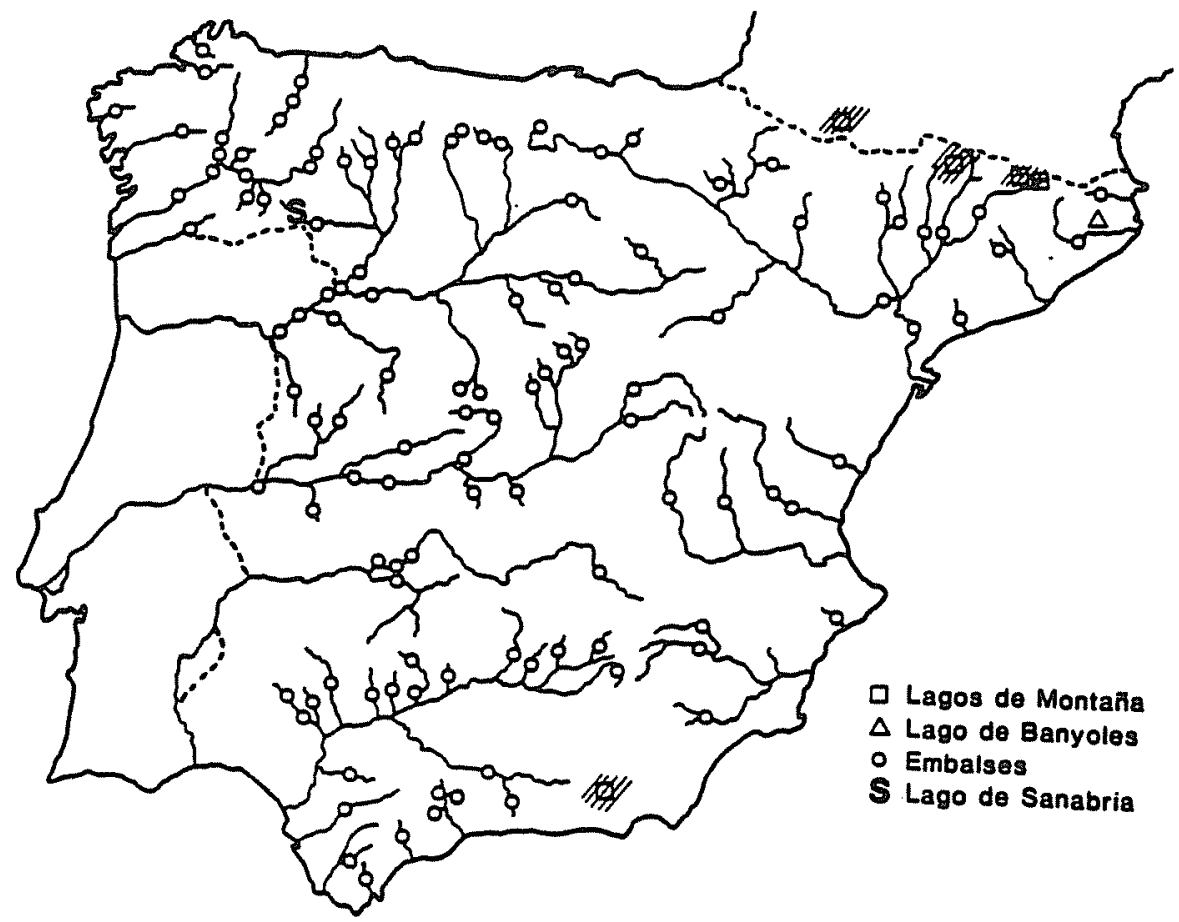

Fig. 1: Situación de los lagos y embalses donde se ha estudiado el bentos en España. Se indica también la situación de los lagos de Neouville (en Francia) estudiados por Laville (1972). 


\section{LAS COMUNIDADES MAS REPRESENTATIVAS.}

Los organismos que han recibido mas atención en los estudios bentónicos de sustrato blando de España son los macrobentónicos, especialmente los oligoquetos y los quironómidos. A los demás componentes bentónicos (hidrácaros, nematodos, ostrácodos, briozoos, otros dípteros) se les ha deparado una atención mas escasa proporcional a su poca densidad o a que su estima se ha hecho después del filtrado con redes de 150 o 250 micras que no permiten la investigación de la dinámica de sus poblaciones. En este momento la lista mas completa es la realizada por Rieradevall (1991) en el lago de Banyoles que incluye, 4 hidrácaros, 8 ostrácodos, 4 nematodos, 9 oligoquetos, 22 quironómidos, 5 quidóridos, 5 copépodos además de bivalvos, cladóceros, gammáridos y efemerópteros hasta llegar a un total de 66 especies. Existen algunas citas de nematodos de los embalses españoles (Prat, 1980) donde lo que es mas conocido son los oligoquetos (Martinez-Ansemil \& Prat 1984; Real \& Prat 1991; Prat et al en prensa) y los quironómidos (Prat 1979a, 1980a, 1980b). En total hasta la actualidad se han citado 92 especies considerando las conocidas del lago de Banyoles, del lago de Sanabria y de los embalses españoles. Esta lista se vera aumentado por nuevas citas en el futuro si se investigan los lagos de montaña o los humedales y si se estudia con mayor atención la meiofauna.

Como rasgos característicos de las comunidades bentónicas de los ambientes lénticos españoles, destacamos la presencia de algunos quironómidos no muy abundantes en otros lagos y embalses europeos, como Microchironomus tener, o Cladopelma virescens, que por los datos de la literatura parecen ser propios de zonas de aguas mineralizadas. Asímismo es de destacar que una vez clasíficados por sus cromosomas los ejemplares del género Chironomus tanto del lago de Banyoles como de algunos embalses (Rieradevall \& Prat 1989 y datos inéditos), solo tres especies parecen habitar nuestros fondos, $C h$. plumosus, $C h$. bernensis y $C h$. nuditarsis. Esto constituye una cierta novedad con respecto a muchos lagos europeos donde existen otras especies (principalmente el conocido Ch. anthracinus). Es curioso resaltar la coincidencia de especies de este género entre el lago de Banyoles, nuestros embalses, el lago Balaton (Hungria) y el lago de Neusierdersee (Austria), todos ellos caracterizados por sus aguas mineralizadas así como el Wolhensee (Suiza) donde se encontró por primera vez el Ch. bernensis. También la presencia de Potamothix heuscheri, un tubificido que parece muy relacionado con ambientes donde el sulfhídrico es abundante, es destacable dentro de la lista de especies por su frecuencia y por no ser habitual en el centro de Europa. De hecho nuestra lista de especies se aproxima mucho a la de algunos lagos y embalses italianos (Bazzanti, 1983; Seminara \& Bazzanti, 1988). Una lista completa de los organismos encontrados hasta el momento se presenta en un anejo a este trabajo. Esta lista se ha elaborado con los datos de la literatura, y algunos de inéditos propios o facilitados por M. Rieradevall y M. Real.

\section{RELACIONES CON LOS FACTORES DEL MEDIO.}

No se han realizado hasta el momento experimentos que demuestren claramente la importancia de los diferentes factores en el establecimiento y mantenimiento de una comunidad determinada, pero tenemos datos relevantes sobre la importancia de diferentes factores.

\section{El oxígeno como factor clave.}

Es bien conocido de los estudios bentónicos la importancia del oxígeno en determinar la composición de la comunidad desde los trabajos de Walshe (1950) y Jonasson (1972). Los datos que teniamos de los embalses así lo indicaban y han quedado ampliamente confirmados por el estudio del lago de Banyoles, donde la 
variedad y abundancia de la fauna depende de lo largo que sea el periodo de anoxia cerca del sustrato. En Banyoles existen cubetas con la sola presencia de Chaoborus, cuando la anoxia es total y prolongada (la III con una meromixis permanente) hasta la presencia de una comunidad altamente diversificada con 20 quironómidos diferentes y especies de oligoquetos cuando no hay déficit de oxigeno o este es inferior a 1 mes, pasando por situaciones intermedias en la que una anoxia de 6 meses reduce la comunidad a 2 especies de quironómidos y 1 de oligoquetos (tabla I) (Rieradevall \& Prat, 1991). La facilidad de dispersión de las larvas o las puestas de adultos en los quironómidos y algunos oligoquetos estan en la base de la recolonización rápida de los fondos donde la fauna es aniquilada por las duras condiciones del medio.

Aunque no poseemos datos concluyentes, la presencia de sulfhídrico debe jugar un papel preponderante en la eliminación de la macrofauna, pues en condiciones de anoxia profunda a lo largo de diversos meses en muchos lagos europeos no se produce la muerte de las larvas de quironómidos. Así lo demuestran diversos autores entre los que citaremos el trabajo de Jonasson (1972) y los experimentos de Frank (1983) que mostraron como algunas especies tenian metabolismo anaeróbico. Recientes experimentos de Heinis \& Crommentuijn (1989) han demostrado la importancia del oxígeno como factor clave y como las diferentes especies tienen tolerancias a diferentes concentraciones lo que se manifiesta en su comportamiento respiratorio y alimentario. En España la abundancia de sulfato en muchas cuencas daria al oxigeno un papel aun mas importante que el ejercido en otras zonas (Finlandia, Suecia), donde la anoxia no supone necesariamente la presencia de importantes cantidades de sulfhídrico en el agua intersticial y por lo tanto la toxicidad es menor para los organismos.

Tabla I. Densidad de los principales grupos de macrobentos en dos lagos (Banyoles y Sanabria) y un embalse (Conde de Guadalhorce) españoles en tres transectos a diferentes profundidades. Se indica la granulometria de cada estación y los meses en un ciclo anual con poco oxígeno cerca del fondo. En el caso de los dos lagos son promedios anuales, en el caso del embalse son datos de un solo muestreo. Las cubetas solo se indican en el lago de Banyoles según la nomenclatura en Rieradevall \& Prat (1991). En todos los casos son medias de tres réplicas (Datos de Rieradevall \& Prat, 1991 e inéditos).

\begin{tabular}{|c|c|c|c|c|c|c|c|c|c|c|c|}
\hline \multirow{3}{*}{$\begin{array}{l}\text { GUADAL } \\
\text { Cubeta } \\
\text { Protundidad } \\
\text { Meses O2 <1ppm } \\
\text { Granulometria \% } \\
0,2-2 \mathrm{~mm} \\
0,05-0,2 \mathrm{~mm} \\
0,02-0,05 \mathrm{~mm} \\
0,002-0,02 \mathrm{~mm} \\
<0,002 \mathrm{~mm} \text {. } \\
\text { Macrobentos (ind/m2) }\end{array}$} & \multicolumn{6}{|c|}{ LAGO DE BANYOLES } & \multicolumn{3}{|c|}{ LAGO DE SANABAIA } & \multicolumn{2}{|c|}{ CONDE } \\
\hline & $\begin{array}{r}\text { } \\
20 \\
12\end{array}$ & $\begin{array}{r}N \\
14 \\
6\end{array}$ & $\begin{array}{r}1 \\
20 \\
1\end{array}$ & $\begin{array}{c}12^{1} \\
1\end{array}$ & $\begin{array}{l}1 \\
5 \\
0\end{array}$ & $\begin{array}{r}39 \\
0\end{array}$ & $\begin{array}{r}13 \\
0\end{array}$ & $\begin{array}{r}7 ' 5 \\
0\end{array}$ & 21,5 & 10 & 5 \\
\hline & $\begin{array}{r}0 \\
0 \\
2 \\
71 \\
27 \\
2)\end{array}$ & $\begin{array}{r}0 \\
0 \\
1 \\
68 \\
31\end{array}$ & $\begin{array}{r}0 \\
0 \\
1 \\
50 \\
49\end{array}$ & $\begin{array}{r}0 \\
0 \\
7 \\
64 \\
29\end{array}$ & $\begin{array}{r}0 \\
6 \\
3 \\
67 \\
24\end{array}$ & $\begin{array}{r}0 \\
8 \\
25 \\
49 \\
19\end{array}$ & $\begin{array}{r}0 \\
49 \\
29 \\
18 \\
11\end{array}$ & $\begin{array}{r}82 \\
13 \\
2 \\
1 \\
1\end{array}$ & $\begin{array}{r}0 \\
0 \\
6 \\
29 \\
65\end{array}$ & $\begin{array}{r}0 \\
1 \\
10 \\
29 \\
60\end{array}$ & $\begin{array}{r}0 \\
3 \\
29 \\
27 \\
41\end{array}$ \\
\hline $\begin{array}{l}\text { Oligoquetos } \\
\text { Quironómidos } \\
\text { Chaobonus } \\
\text { Elemerópteros } \\
\text { Otros }\end{array}$ & $\begin{array}{r}0 \\
0 \\
465 \\
0 \\
0\end{array}$ & $\begin{array}{r}8 \\
474 \\
1843 \\
0 \\
2\end{array}$ & $\begin{array}{r}1303 \\
438 \\
239 \\
0 \\
62\end{array}$ & $\begin{array}{r}1102 \\
2584 \\
267 \\
0 \\
241\end{array}$ & $\begin{array}{r}850 \\
9178 \\
33 \\
8 \\
371\end{array}$ & $\begin{array}{r}4888 \\
859 \\
0 \\
0 \\
29\end{array}$ & $\begin{array}{r}1103 \\
1070 \\
0 \\
0 \\
259\end{array}$ & $\begin{array}{r}143 \\
3542 \\
0 \\
50 \\
47\end{array}$ & $\begin{array}{r}575 \\
867 \\
0 \\
0 \\
25\end{array}$ & $\begin{array}{r}25 \\
6637 \\
0 \\
0 \\
0\end{array}$ & $\begin{array}{r}25 \\
9514 \\
0 \\
0 \\
0\end{array}$ \\
\hline
\end{tabular}

\section{La temperatura}

La temperatura se perfila también como un factor importante en determinar la importancia de las especies predominantes. Así en el lago de Sanabria, el fondo se halla dominado por quironómidos de la especie Micropsectra contracta, una fauna similar a la del lago de Constanza, el de Ginebra o algunos lagos alpinos alemanes (Prat, 1980b). Temperaturas muy bajas en invierno $\left(4^{\circ} \mathrm{C}\right)$ y relativamente bajas en la época de mezcla junto a un contenido bajo de sales (conductividad de 15 microsiemens y casí no hay sulfato) asi como oxígeno siempre elevado cerca del fondo propician esta situación (Vega et al., en prensa). Solo en un embalse español se encontró en los años 70 algo similar. Era también uno de aguas frias y bien oxigenadas en su momento (embalse de Porma en León) situado relativamente cerca de Sanabria. 
De lo poco que conocemos de los lagos pirenaicos españoles, podemos decir que solo a una cierta altitud hemos encontrado comunidades dominadas por el quironómido Heterotrissocladius, género característico de los lagos ultraoligotróficos nórdicos o canadienses. Las comunidades de las lagunas de Sierra Nevada, a tenor de las exuvias encontradas (Laville \& Vlilchez, 1986), son también típicas de los lagos ultraoligotróficos frios. La temperatura, pues, parece ser un factor directamente implicado en la selección de las especies que colonizan los fondos blandos de nuestros lagos y embalses en las zonas de altitud superior a $1500 \mathrm{~m}$ (aprox.) en el norte del pais (con la excepción de Sanabria a $1000 \mathrm{~m}$. pero en una zona muy fria) y más de $2000 \mathrm{~m}$ en Sierra Nevada, lo que acentua el carácter relicto de la fauna presente en estos puntos.

\section{Textura del sedimento.}

Otro factor muy importante es la textura del sedimento. Los únicos datos que poseemos al respecto por el momento son los de los lagos de Banyoles y Sanabria. En los dos lagos las zonas sublitorales, situadas en lugares con cierta pendiente, tienen una granulometria con relativa abundancia de fracciones groseras, mas claro en Sanabria que en Banyoles en la tabla I, aunque en Banyoles existen zonas sublitorales con una granulometria mas grosera. En estas condiciones los oligoquetos disminuyen su densidad y dominan otras especies, principalmente los quironómidos con presencia de géneros mas escasos en las zonas profundas (Rieradevall \&. Prat; 1991). También con el mayor tamaño de grano (y la menor profundidad) se diversifica la fauna con la presencia de otros grupos además de quironómidos y oligoquetos como ceratopogónidos y algunos efemerópteros (Ephemera, Caenis en los dos lagos) y los Echinogammarus pungens de Banyoles. En los embalses estos elementos son mucho mas escasos cuando la fluctuación es importante (tabla I) aunque pueden ser importantes si la fluctuación es menor como en el caso del embalse de Orellana, tercero de una serie en el rio Guadiana (Prat et al 1991, tabla II).

\section{Alimenteción.}

Otro aspecto muy importante es como se organiza la comunidad en el aspecto trófico. Los organismos bentónicos se alimentan de lo que proviene de la capa de agua que existe sobre ellos. En los lagos muy productivos y grandes, es de esperar que una gran parte de la alimentación dependa de las algas que sedimentan. Así lo demostró Jonasson (1972)y la dependencia de la producción secundaria de la primaria en el lago Esrom ha sido puesta de manifiesto varias veces (Jonasson et al 1990) y tomada como ejemplo en muchos manuales.

Sin embargo en los lagos oligotróficos pequeños como los nuestros (Sanabria, Banyoles), los aportes que reciben los fondos pueden tener un origen mas dependiente de su cuenca adyacente (materiales alóctonos como polen o hojas) o bien de los producidos en el lago pero por via detrítica (macrófitos), que no de los generados en el epilimnion del lago (fitoplancton). Por ello la comunidad puede organizarse de modo muy diferente a lo descrito clásicamente. Los detritívoros de pequeño tamaño (nematodos, ostrácodos, oligoquetos) pueden verse así favorecidos y ello provocar que los pequeños microcarnivoros (quironómidos, especialmente los géneros Cladopelma, Microchironomus o Procladius) se vean especialmente favorecidos frente a los fitófagos como Chironomus. Ello explicaria las bajas densidades de animales filtradores en lagos pequeños y oligotróficos como el de Banyoles o el de Sanabria y la mayor dominancia de los oligoquetos en sus partes mas profundas junto a los Procladius: (ver Fig. 2)

Esta situación se repite en los embalses donde los aportes alóctonos son siempre abundantes por el cambio de nivel de sus orillas, la rápida renovación de sus aguas y la erosión de sus cuencas. Esto cuadra bien con la dominancia de los Procladius en la mayoría de embalses (Prat, 1980). En el caso de los embalse, pues, la fluctuación de las orillas que origina una sedimentación muy rápida en sus fondos es un factor muy importante en la presencia o no de muchas especies. 


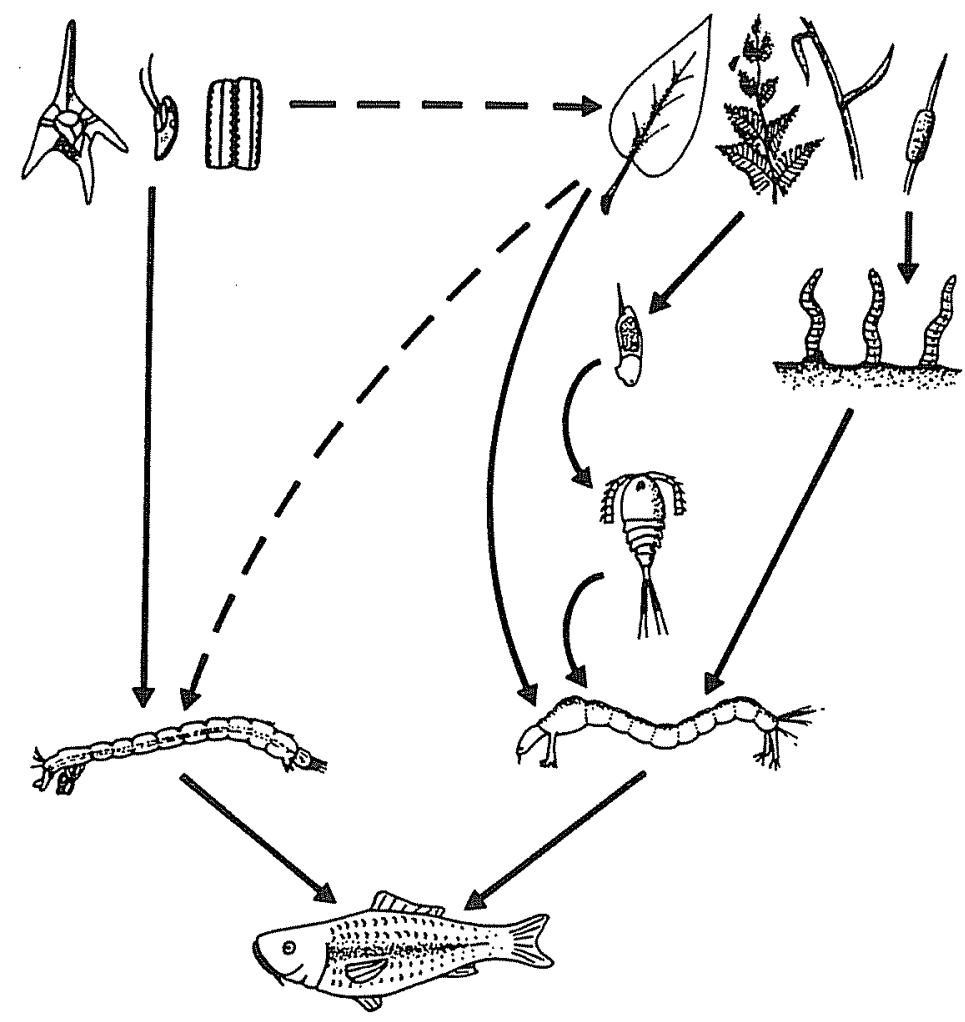

Fig. 2: Las dos rutas principales de alimentación de los organismos bentónicos. La de la izquierda favorece a los organismos fitófagos como Chironomus, mientras que la via heterotrófica (derecha) favoreceria a los detritivoros y como resultado aumentaria la presencia de quironómidos microcarnívoros como Procladius. Tanto en el lago de Banyoles como en Sanabria la via detritivora parece ser la mas favorecida. Las flechas discontinuas quieren indicar las conexiones que pueden existir entre las dos vias.

\section{Importancia de la profundidad.}

Las comunidades de las zonas profundas son diferentes a las de las zonas o lagos menos profundos como ya señalamos en nuestro trabajo inicial (Prat, 1979b,1980b) y como hemos visto en Banyoles (Rieradevall \& Prat, 1991) o en Sanabria. A mas profundidad disminuye la presencia o abundancia de muchas especies y aumenta, en general, la abundancia de los oligoquetos con respecto a los quironómidos. Esto ocurre tanto en un lago o embalse en concreto comparando la fauna sublitoral con la profunda (Sanabria, Banyoles, embalses del Guadalhorce, tabla I) como comparando diferentes situaciones de profundidad máxima distinta (embalses), véase por ejemplo la situación en los tres embalses correlativos del Guadiana (tabla II). De las tablas se deduce también que la densidad total del bentos disminuye a medida que aumenta la profundidad. Esto no conlleva siempre necesariamente en una menor variedad de especies tal como se ve en Banyoles donde todas las especies encontradas a $5 \mathrm{~m}$. de oligoquetos y quironómidos se encontraron también a $20 \mathrm{~m}$ a lo largo del ciclo anual, aunque desaparecían otros grupos.

Una hipótesis plausible de estas diferencias, cuando no hay déficit de oxígeno que explica la mayoría de los casos, es relacionar estas diferencias con el estado del 
material que sedimenta hacia el fondo. Es de suponer que cuanta mas profundidad, menos fresco puede llegar el material producido en el propio lago (fitoplancton) y por lo tanto la proporción de material procesado por la via detrítica aumenta. Esto cuadra perfectamente con la mayor abundancia en promedio de oligoquetos y Procladius a mayor profundidad. La situación contraria no es generalizable y embalses poco profundos con producción primaria elevada pueden presentar gran número de fitófagos (Chironomus) en algunos casos y en otros no. Otros factores propician esta abundancia o no aunque este es un punto que requiere una mayor profundización y debe investigarse individualmente en cada embalse.

\section{Efecto de las fluctuaciones de nivel.}

En los embalses los cambios de nivel son muy importantes, tanto porqué impiden la presencia de vegetación macrofítica y así se reducen las posibilidades de colonización por parte de muchos animales, como por el arrastre de materiales hacia el fondo que supone la fluctuación.

Ya se apuntaron algunos hechos como estos en nuestros primeros estudios (Prat, :1980b) que luegon se han confirmado con otros mas recientes (Prat et al, 1991). En promedio, la variedad y abundancia de la zona que queda por encima de la termoclina es mayor que la de la zona profunda aunque con diferencias mínimas en algunas épocas del año como se vio en el estudio que hicimos de tres embalses de la cuenca del Guadiana (Prat el al. 1991). En este mismo estudio, se vio claramente una diferencia de variedad y abundancia entre las estaciones del primer embalse (sujeto a mayor fluctuación) y las del último, mas constante en su nivel (tabla II). En este sentido nos falta todavia un buen estudio de las faunas de la zona litoral de alguno de nuestros lagos (especialmente Banyoles) para poder conocer la situación mas ideal que resultaria de una nula fluctuación del nivel del agua. Un ejemplo interesante seria comparar el litoral del lago de Sanabria (con una fluctuación de casi un metro por año, pero regular año tras año) con algunos de los embalses que existen en la misma zona sometidos a mayor fluctuación. Es una lástima que los estudios de impacto que se realizan en muchos de nuestros embalses no puedan ser utilizados como un test en este sentido. Ni siquiera es facil conseguir los escasos datos que sobre ellos se tienen del bentos.

Tabla II. Importancia de la profundidad, la concentración de oxígeno cerca del fondo y la fluctuación del nivel del agua en tres embalses correlativos del rio Guadiana. Orellana es el situado aguas abajo y las estaciones 1 son las situadas mas cerca de la presa. Quir/Olig indica el resultado de dividir la densidad media de los quironómidos por la de oligoquetos. La densidad es un promedio anual de 6 muestras. $\mathrm{P}=$ Procladius. $\mathrm{Ch}=$ Chironomus. (Datos de Prat et al 1991)

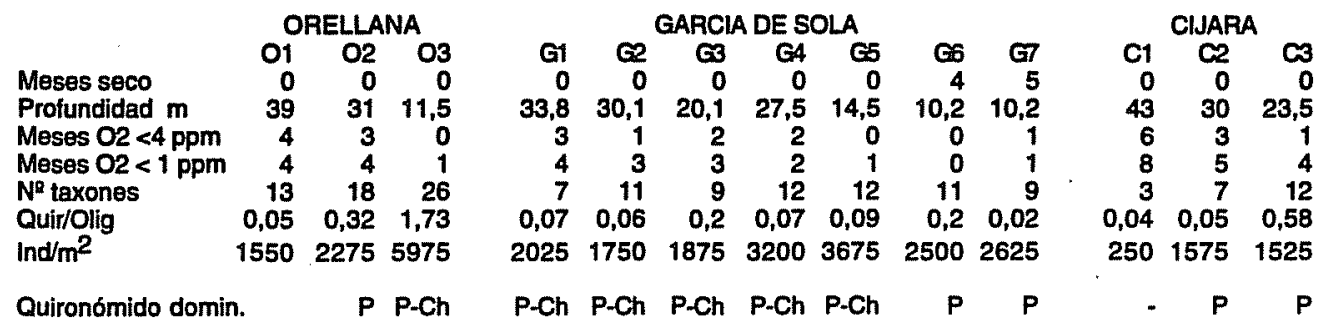

\section{COLONIZACION Y SUCESION.}

En los experimentos de colonización realizados por Prat (1980c) se demostró claramente que después de una fase inicial de colonización rápida en la que dominaban los quironómidos de tipo fitófago, se hacían mas abundantes los oligoquetos y algunos quironómidos microcarnívoros (Microchironomus). Esto sucedía si las condiciones de oxigenación eran buenas, pues sino la comunidad bentónica quedaba 
reducida a los dipteros caobóridos (Chaoborus flavicans) por la posibilidad que tienen de migrar fuera de la zona anóxica durante un cierto periodo del dia.

En aquel estudio se mostró como en un tiempo relativamente corto (menos de un año), la comunidad del embalse, de un fondo blando y que no fluctua de nivel, se estructuraba de una forma relativamente compleja. De hecho se llegaba a una estructura similar a la de la zona sublitoral del lago de Banyoles. A lo largo del proceso de recolonización, la riqueza específica y la diversidad aumentó de forma considerable. Sobre este proceso es de suponer que en años venideros actuarian de forma mas clara los factores propios del embalse (temperatura, producción primaria) o los bióticos (predación, competencia), dando un conjunto de fluctuaciones en la diversidad y producción de la comunidad similares a los de otros lagos. El factor a resaltar es la rapidez de colonización por diferentes elementos debido a la facilidad de dispersión y la resistencia a condiciones adversas típico de las especies bentónicas por ser organismos ya "preseleccionados" a resistir el estrés de los ambientes que habitualmente colonizan (la mayoría son especies pioneras con ciclos de vida corto).

\section{TIPOLOGIA.}

El bentos de lagos y embalses ha sido siempre considerado como un buen indicador de las características globales de un lago, especialmente de su eutrofia. EI sistema simple inicial desarrollado por Thienemann y mas tarde por Brundin (1958) fue perfeccionado de forma mas sutil por Saether (1979) mas recientemente. Este sistema incluye 15 clases de lagos tipificados segun las comunidades de quironómidos presentes y parece funcionar bien para los grandes lagos americanos y del norte de Europa.

En España las especies mas estenotermas de agua fria no son abundantes y por ello los lagos mas oligotróficos en el sistema de Saether (con Heterotrissocladius) se hallan presentes solo en las partes mas altas del Pirineo y de Sierra Nevada, con lo que 5 clases quedan ya muy poco representadas en la península. Los lagos oligotróficos de tipo Micropsectra parecen reducirse en España al de Sanabria y a algunas lagunas de montaña, con lo que 5 clases mas quedan fuera de una posible tipificación de los embalses españoles siguiendo el sistema de Saether. Así para los embalses españoles y el lago de Banyoles solo restarían 5 clases, consideradas todas por Saether como lagos eutróficos.

Utilizando este sistema, todos los embalses del.Duero y del Guadiana y el lago de Banyoles quedan situados de forma dispersa en las cinco clases que restan sin seguir la pauta sugerida pos Saether (Fig. 3), lo que significaria que ni el fósforo ni la clorofila (la mayor o menor producción en síntesis) son el factor clave de la tipología de comunidades bentónicas en los ambientes lénticos españoles. Ello es debido, sin duda, a que la temperatura de forma directa e indirecta juega un papel trascendente y modifica la importancia de la producción como elemento clave de la tipología. Directa al impedir la presencia de las especies mas estenotérmicas e indirecta porque aumenta el periodo de estratificación y por ello el tiempo en que el hipolimnion queda aislado del epilimnion y asi se puede agotar el oxígeno de forma mas rápida y que permanezca el hipolimnion anóxico mas tiempo con producciones primarias relativamente bajas. Junto a ello la mayor mineralización del agua parece actuar de una forma sinérgica, favoreciendo la presencia de tóxicos que eliminan facilmente a los organismos, especialmente cuando abundan los sulfatos. El oxígeno y el sulfídrico se convierten de esta forma en los factores claves para una tipología, mas que la relación con las características tróficas del lago como la clorofila o el fósforo. En los embalses, existe además una gradación norte sur, de forma que los embalses del Guadalquivir tienen aun mas exagerado este proceso y en muchos de ellos la fauna profunda desparece en los peridos de estratificación (Real datos inéditos). 

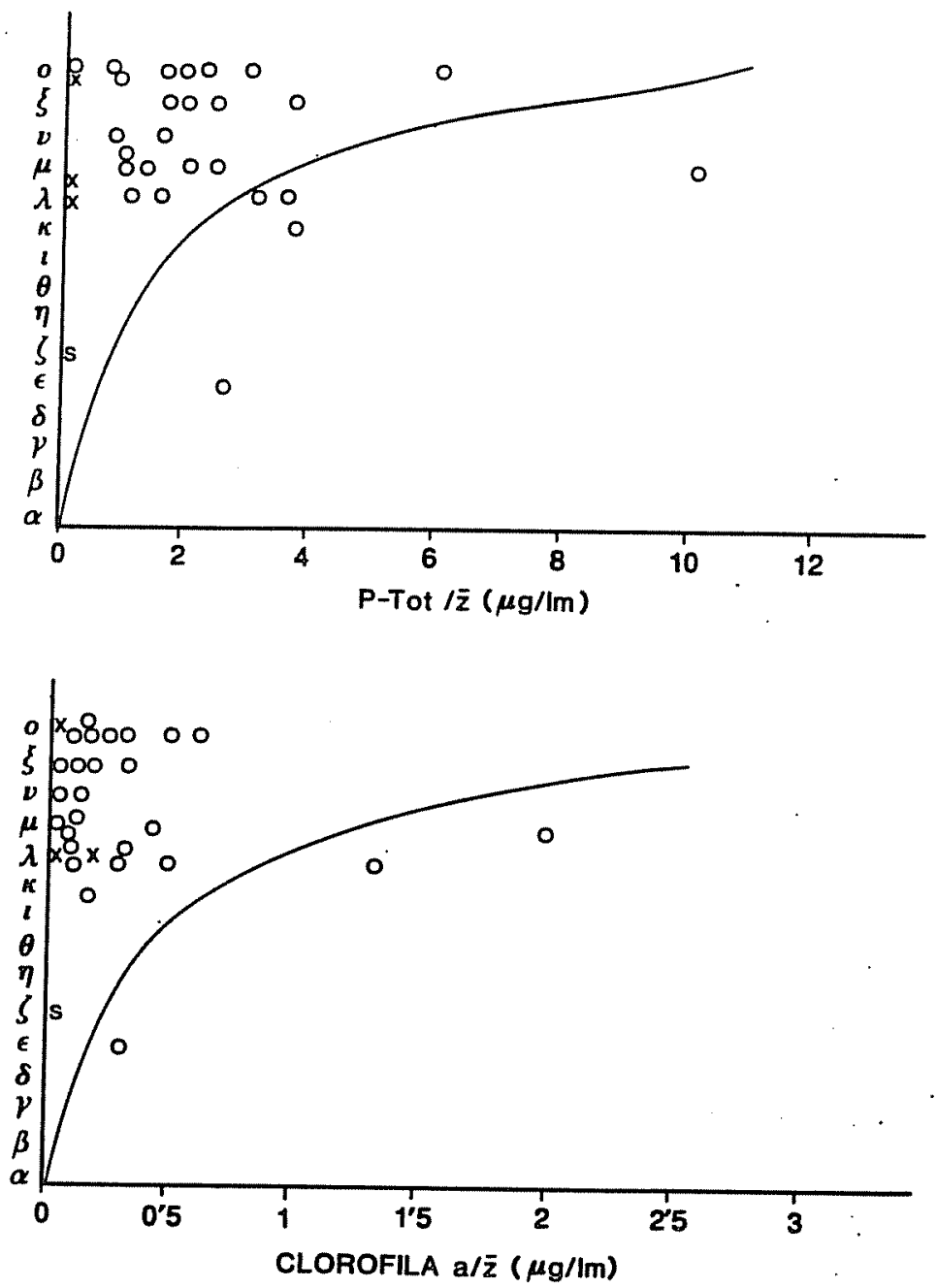

Fig. 3: Situación de algunos lagos y embalses españoles en el sistema tipológico ideado por Saether $\mathbf{S}=$ Sanabria. $x=$ Diferentes cubetas del lago de Banyoles. $0=$ Embalses de la cuenca del Duero. No existe la relación comunidad-eutrofia preconizada por el sistema de Saether, con lo que este no parece válido para los lagos y embalses españoles.

\section{IDEAS PARA UN MODELO CONCEPTUAL.}

La fauna de nuestros lagos y embalses parece pues afectada de forma profunda por algunos factores de estrés que actuan de forma algo diferente en su importancia respecto a los lagos europeos como resultado de nuestro clima que favorece un periodo mas largo de estratificación. 
El factor mas importante de estrés son las condiciones que posibilitan que falte oxígeno cerca del fondo. Si esto se acompaña de la presencia de sulfatos hace que el fondo este desprovisto de organismos o con la sola presencia de los Chaoborus. Esto vale tanto para lagos (Banyoles) como para embalses (tabla I).

La menor mineralización del agua o un perido mas corto de anoxia, posibilita la presencia en el fondo de algunos organismos resistentes a presiones parciales bajas de oxígeno, especialmente los tubifícidos (con $P$. heuscheri como elemento mas relevante) y los quironómidos del género Chironomus. Estos se ven favorecidos en las zonas con poca profundidad y viceversa los oligoquetos en las de mayor profundidad. La via detritivora y una tasa de sedimentación mas elevada favorecen asímismo la mayor importancia de los oligoquetos respecto a los quironómidos.

En condiciones mejores de oxígeno, si la fluctuación de las orillas es mínima, la importancia de la procedencia del alimento parece ser la clave del tipo de comunidad que se encuentra. La via detritívora se da mas en los lagos pequeños con cuencas forestadas o algunos macrófitos en las orillas (Banyoles, Sanabria) o en embalses de situación similar (Galicia). En estos casos los oligoquetos siguen siendo dominantes y entre los quironómidos los mas abundantes son los del género Procladius. (Fig. 2).

Cuando la fluctuación de las orillas es importante, el aporte de materiales alóctonos y sedimentos al fondo es un factor clave. En estas condiciones la fauna bentónica se hace escasa y dominada por oligoquetos. Entre los quironómidos los Procladius, por su movilidad, son los que mas rapida y abundantemente se encuentran en el fondo. De aqui la importancia de la asociación oligoquetos-Procladius presente en una gran mayoría de embalses por la importancia de las fluctuaciones y la entrada de materiales alóctonos.

En igualdad de condiciones de fluctuación y con eutrofia creciente es de esperar la sucesión Tanytarsus-Stictochironomus-Chironomus. Además estas comunidades pueden variar a lo largo del año; en invierno-primavera y cuanto mas al sur, los quironómidos pueden ser presentes y abundantes en los embalses de menor profundidad. En verano con la anoxia y la presencia de sulfhídrico estas comunidades pueden desaparecer. El eje temporal tiene pues un peso muy importante y las zonas litorales o sublitorales con oxígeno se convierten en reservorios para la posterior recolonización de las zonas profundas.

Con estos factores es mas fácil comprender como puede estructurarse la fauna bentónica. Algunas relaciones entre el tamaño de los oligoquetos con factores de la cuenca (erosión, características químicas del sedimento) pueden dar en el futuro mas detalle a este modelo (Real et al. 1992) cuya precisión y peculiaridades deben buscarse en cada caso sin que sea posible, por ahora, desarrollar un sistema mas predictible.

\section{AGRADECIMIENTOS.}

Este trabajo es una suma de muchas ayudas. Sin duda sin la intensa colaboración que he tenido en estos últimos años por parte de Maria Rieradevall, Enrique MartinezAnsemil y Montserrat Real no habria desarrollado la mayoria de las ideas expresadas aqui. En diferentes fases han colaborado también Josefina Sanz, Isabel Muñoz, Carles Ibañez y algunos estudiantes de los cursos de Limnología. Datos inéditos me han sido proporcionados gentilmente por Limnos S.A. y M. A. Puig. A Maria también le debo muchos comentarios y horas de discusión y apoyo que han contribuido a mejorar algunos aspectos de mi trabajo. Diversos contratos con la CAYCIT, Limnos y otras instituciones han dado el soporte económico a nuestro trabajo. 
BIBLIOGRAFIA

Bazzanti M. 1983. Composition and diversity of the profundal macrozoobenthic community in the polluted lake Nemi (Central Italy). 1979-80. Acta Oecol. oecol. appl. 4(3): 211-220.

Brundin, L. 1958. The bottom faunistical lake type system and its application to the southem hemisphere: Moreoever a theory of glacial as a factor of productivity in lakes and oceans. Verh. Internat. Verein. Limnol., 13: 288-297.

Frank, C. 1983. Ecology, production and anaerobic metabolism of Chironomus plumosus L. in a shallow lake II. Anaerobic metabolism. Arch. Hydrobiol., 96: 354-362.

Heinis, F. \& Crommentjuin, T. 1989. The natural habitat of the deposit feeding chironomid larvaeStictochironomus histrio (Fab) and Chironomus anthracinus Zett. in relation to their responses to changing oxygen concentrations. Acta Biol, Debrec. Oecol. Hung., 3: 141-146.

Jonasson, P.M. 1972. Ecology and production of the profundal benthos. Qikos, suppl., 14: 1-148.

Jonasson, P.M.; C. Lindegaard, C.; P.C. Dall.; K. Hamburger. \& H. Adalsteisson, 1990. Ecosystem studies on temperate lake Esrom and the subartic lakes Myvatn and Thingvallavatn. Limnologica (Berlin) 20: 259-266.

Laville, H. 1972. Recherches sur les chironomides (Dipt) des lacs du massif de Néouvielle (Hautes-Pyrénées). Première partie: Systématique, Ecologie, Phénologie. Ann. Limnol. 7: 173-332.

Laville, H. \& A. Vlilchez, 1986. Les Chironomidés (Diptera) de quelques "lagunas" de haute altitude de la Sierra Nevada (Granada, Espagne). Annls. Limnol. 22(1): 53-63.

Limnos S.A. 1989. Estudio diagnóstico de la calidad de las aguas embalsadas de la comunidad de Castilla y León: Determinación y previsión de impactos, propuesta de corrección y red de viqilancia. 3 tomos.

Malo, J. 1987. Evolución de las poblaciones planctónicas y de quironómidos en el embalse de Talave (Rio Segura, SE España). Tesis de Licenciatura. Univ. Murcia. $196 \mathrm{pp}$.

Martinez-Ansemil, E. \& N. Prat. 1984. Oligochaeta from profundal zones of Spanish reservoirs. Hydrobiologia, 115: 223-230.

Margalef; R. D. Planas; J. Armengol; A. Vidal; N. Prat; A. Guiset; J. Toja \& M. Estrada1976 Limnologia de los embalses españoles. Public. Minist. Obras Públicas. $\mathrm{n}^{2} .123$.

Molina, C. 1989. Dinámica de la población de Anguilla anguilla en una laguna artificial asilvestrada (Alicante, SE España). Tesina licenciatura. 123 págs.

Prat, N. 1976. Fauna bentónica de los embalses españoles. Resúmenes II Asamblea Nacional de Geodesia y Geofísica. págs. 2041-2054.

Prat, N. 1978. Benthic typology of Spanish reservoirs. Verh. Internat. Verein. Limnol, 20: 1647-1651.

Prat, N. 1979a. Quironómidos de los embalses españoles (I). Graellsia, XXXIIl: 37-96.

Prat, N. 1979b. Fauna marginal de los embalses españoles. Misc. Zoologica, 5: 149-160.

Prat, N. 1980a. Quironómidos de los embalses españoles (II). Graellsia, XXXIV: 59-119.

Prat, N. 1980b. Bentos de los embalses españoles. Oecol. aquat., 4: 3-43.

Prat, N. 1980c. Benthic population dynamics in artificial samplers in an Spanish reservoir. IN: Chironomidae: Ecology, Systematics and Physiology (D.A. Murray ed.). págs, 239-246. Pergamon Press. Oxford and New York.

Prat, N. \& V Daroca, 1983. Eutrophication processes of Spanish reservoirs as revealed by biological records in profundal sediments. Hydrobiologia, 103: 153-158.

Prat, N., J. Sanz \& E. Martinez-Ansemil, 1991. El bentos litoral y profundo de una cadena de tres embalses españoles del rio Guadiana. Limnetica, 133-152.

Prat, N. \& M. Rieradevall, en prensa. Life cycle and production of Cladopelma virescens

(Mg.) (Dipt. Chironomidae) in lake of Banyoles (NE Spain). Neth. J. Aquat. Ecol.,26 (2-4).

Real, M. \& N. Prat, N. 1991. Changes in the benthos of five Spanish reservoirs in the last 
15 years. Verh. Intemat. Verein. Limnol, 1377-1381.

Real, M.; F. Sabater \& J.A. Morgui, 1992. Disturbances in the Ebro basin reservoir (NE Spain) reflected by relationships among sediment, water and benthic fauna. Hydrobiologia, 235/236: 363-374.

Rieradevall, M. \& N. Prat, N.1989. Chironomidae from profundal samples of Banyoles lake. Acta Biol. Debr. Oecol. Hung., 3: 267-274.

Rieradevall, M. \& N. Prat, N. 1991. Benthic fauna of Banyoles lake. Verh. Intemat. Verein. Limnol., 24: 1020-1023.

Rieradevall, M; C. Ibañez, I. Muñoz, M. Real \& N. Prat en prensa. Grupos de macroinvertebrados del bentos del lago de Sanabria y su relación con la granulometria del sedimento. Actas VI Congreso Esp. Limnologia. Granada 1991.

Saether, O.A. 1979. Chironomid communities as water quality indicators. Holarct. Ecol., 2: 65-74.

Seminara, M. \& M. Bazzanti, 1988. Trophic level assessment of profundal sediments of the artificial lake Campotosto (Central ltaly), using midge larval community (Dipt. Chir.). Hydrobiol. Bullt., 22(2): 183-193.

Vega, J.C.; C. de Hoyos \& J. Aldosoro, en prensa. Limnologia del Lago de Sanabria. Publicaciones de la Consejeria de Medio Ambiente de la Junta de Castilla y León.

Walshe, B. M. 1950. The function of haemoglobin in Chironomus plumosus under natural conditions. J. exp. Biol.s 27: 73-95.

APENDICE. Lista de especies encontradas hasta el momento en el bentos de los lagos y embalses españoles. $B=$ Banyoles, $S=$ Sanabria, $E=$ Embalses. Datos propios e inéditos de M. Rieradevall y M. Real.

NEMATODA

Daptonema dubium, $B$

Dorylaimus stagnalis, E, B

Tobrilus sp. (T. gracilis), E

OLIGOCHAETA

Dero digitata, $B, E$

Aulodrilus pluriseta, $E$

Aulodrilus pigeti, $B, E$

Branchiura sowerbyi, B, E,

Limnodrilus claparedeianus, B, E

L. hoffmeisteri, B, E,

Limnodrilus profundicola, E

L. udekemianus, $\mathbf{E}$

Potamothrix bavaricus, $B, E$

P. hammoniensis, $B, E$,

P. heuscheri, B. E

Tubifex tubifex, $E$

Psammoryctides barbatus, B, E

E. buchholzi, E,

B. appendiculata

BIVALVIA

Pisidium milium, B

Pisidium nitidum, B

llyocryptus sordidus, B, E,
Ironus ignavus, $\mathrm{E}$

Limnomermis bostrychodes, $E$

Mermithis, B

Paranais frici, $E$

Nais pardalis, E

Nais communis, $E$

Nais barbata, $E$.

Nais variabilis, $E$.

Nais pseudoblusa, $\mathrm{E}$.

Ophionais serpentina, E

Pr. menoni, E,

Uncinais uncinata, $E$

Spirosperma ferox, E, S

Haber pyrenaicus, $\mathbf{S}$

Embolocepahlus velutinus, $S$

Bothrionerum vejdowskyanum, $\mathrm{E}$.

Fridericia sp., E.

Pisidium obtusale, B

Pisidium subtruncatum, B 